\title{
Caractérisation des raisonnements des élèves Marocains de 11 à 13 ans dans la résolution de problèmes algébriques
}

\section{Characterization of the reasoning of Moroccan students from 11 to 13 years old in the resolution of algebraic problems}

\author{
Said Abouhanifa ${ }^{1 *}$ \\ ${ }^{1}$ Centre Régional des Métiers de l'Education et de la Formation Casablanca-Settat, Settat, Maroc
}

\begin{abstract}
Résumé. Cette étude vise la caractérisation des différents types de raisonnements des élèves selon leur degré d'analycité et la nature du registre de représentation sémiotique. Pour cela, nous avons administré un questionnaire formé de cinq problèmes de comparaison à un échantillon comportant 518 élèves marocains de 23 classes, qui appartiennent à 19 établissements scolaires de la 6e primaire et du secondaire collégial. Nous avons exploité dans notre cadre d'analyse, la grille développée par Squalli et al, (2020) [1]. Les résultats dégagés laissent apparaître que les élèves de notre échantillon mettent en évidence des catégories de raisonnement analytique et à tendance analytique bien que peu figurées sont également présentes, essentiellement chez les élèves des classes de $6 \mathrm{e}$ année primaire.
\end{abstract}

\begin{abstract}
This study aims at characterizing the different types of reasoning of the students according to their degree of analycity and the nature of the register of semiotic representation. For this purpose, we administered a questionnaire consisting of five comparison problems to a sample of 518 Moroccan students from 23 classes, belonging to 19 schools in the 6th primary and secondary college levels. We used the grid developed by Squalli et al. (2020) [1] in our analytical framework. The results show that the students in our sample display categories of analytical reasoning and analytical tendencies, although not very much is also present, mainly among students in the 6th grade of primary school.
\end{abstract}

\section{Introduction}

\footnotetext{
* Corresponding author: saidabouhanifa@yahoo.fr
} 
Notre problème de départ est de comprendre et analyser les raisonnements des élèves dans la résolution de problèmes algébriques. Cette étude a été réalisée au Maroc. Elle s'inscrit dans la continuité des enquêtes de l'observatoire de la pensée algébrique (OIPA) réalisées au Québec et en France et qui ont porté sur les procédures de résolution de problèmes algébriques de type comparaison chez les élèves avant et après l'introduction de l'algèbre (Marchand et Bednarz,1999, 2000 ; Saboya, Besançon, Martin, Adihou, Squalli et Tremblay, 2014 ; Adihou, Squalli, Saboya, Tremblay et Lapointe, 2015) [2-5]. Elle vise à caractériser les différents types de raisonnements selon leur degré d'analycité (Marchand, 1998) [6] et la nature du registre sémiotique de représentation (Duval 1995) [7]. Pour cela, nous avons administré un questionnaire formé de cinq problèmes à un échantillon comportant 518 élèves marocains de 23 classes, qui appartiennent à 19 établissements scolaires de la 6e primaire et de la première année du secondaire collégial. L'analyse exploite la grille d'analyse développée par Squalli et al. (2020) [1]. Cette grille permet de classer les raisonnements selon trois catégories : 1) raisonnements de type synthétique, où l'élève opère sur des données et des relations connues pour déterminer les valeurs des inconnues, 2) raisonnements à tendance analytique qui ne sont pas synthétiques, où l'élève peut recourir à un registre de représentation non littéral et 3) raisonnements analytiques, l'élève représente les inconnues avec des lettres, utilise ces lettres pour représenter les relations entre les données connues et inconnues du problème et il opère sur les relations et les équations obtenues pour trouver les valeurs des inconnues.

Après avoir présenté la problématique et le cadre d'étude, nous présentons la démarche qui nous a conduit à l'exploitation de notre cadre d'analyse, nous présentons ensuite quelques résultats qui nous ont permis d'identifier les catégories de raisonnements réalisées par les élèves dans la résolution de problèmes de partages inégaux, voire de contribuer à éclairer et enrichir le développement de la pensée algébrique dans le contexte Marocain.

\section{Problématique}

$\mathrm{Au}$ Maroc, les orientations pédagogiques (programmes) du secondaire collégial (2009) envisagent la voie classique des équations pour l'introduction de l'algèbre. Deux facettes sont préconisées à cette entrée à l'algèbre dans les premiers apprentissages du secondaire collégial. La première facette s'occupe des expressions algébriques et la deuxième se consacre à la résolution des équations de premier degré à une inconnue. L'apprentissage du calcul algébrique occupe une place importante dans l'enseignement de l'algèbre, il est mobilisé dans des activités de mathématisation de diverses situations par des expressions algébriques ainsi que dans des activités de résolution de problèmes se ramenant à la résolution d'équations algébriques du premier degré à une inconnue.

L'algèbre a été conçue comme étant un domaine de la discipline mathématique convoitant les règles des opérations sur les nombres et la résolution des équations. Elle évolue plus tard sous une forme d'une doctrine des opérations ensuite des propriétés et des relations sur les objets mathématiques.

L'algèbre est un domaine de l'organisation mathématique qui permet d'exprimer les propriétés des opérations et le traitement des équations et qui aboutit à l'étude des structures algébriques. Selon Lee (1997) [8], il existe une multitude de visions en ce qui concerne la notion d'algèbre. En effet, elle présente six visions différentes qui ressortent d'une recherche où elle a demandé à des enseignants, des didacticiens et des mathématiciens, ce qu'était pour eux l'algèbre. Elle a indiqué qu'il y a l'algèbre en tant que langage, manière de penser, matière scolaire, outil, activité et arithmétique généralisée. Elle conclut par ailleurs qu'il n'existe pas de consensus à ce sujet. Quant à Kaput (1995) [9], l'algèbre peut être décrite selon deux types de discours ; c'est un ensemble d'artefacts culturels tacitement partagés lorsque différentes activités algébriques sont exécutées et d'autre part, comme une manière 
de penser (généralisation, abstraction, justification, etc.). Il distingue encore cinq aspects importants qui concernent la catégorie du raisonnement algébrique (la généralisation et le formalisme algébrique), les sujets mathématiques importants (étude des structures et des systèmes abstraits de l'arithmétique comme les relations et les fonctions) et le langage utilisé (outil de modélisation).

Radford (2006, 2008, 2014) [10-12] présente trois grandes caractéristiques indispensables du développement de la pensée algébrique :

- L'indétermination : la situation avec laquelle l'élève travaille contient des quantités dont les valeurs ne sont pas déterminées (inconnues, variables, les paramètres, etc.) ;

- La dénotation : la capacité à designer des quantités inconnues (symboles, gestes, etc.) ;

- L'analycité : c'est la capacité de raisonner sur les inconnus comme s'ils étaient connus et d'opérer sur ceux-ci.

Le développement de la pensée algébrique dans le contexte de résolution de problèmes est conditionné par le développement du raisonnement analytique, qui consiste à considérer les inconnues, les représenter par des lettres, à opérer sur ces symboles pour former les relations et équations et finalement, à trouver les valeurs des inconnues (Squalli et al, 2020) [1]. Pour Sierpinska (1999) [13], l'algèbre est un produit de la pensée analytique qui nécessite un fonctionnement cognitif spécifique. C'est-à-dire, une pensée qui s'appuie sur des systèmes de représentation externes et conventionnels, permettant l'accès direct avec un objet à partir d'une description verbale.

Plusieurs études (Booth, 1988 ; Chevallard, 1989 ; Kieran, 1994 ; Vlassis \& Demonty, 1999 ; Subramaaiam, 2004) [14-18] ont déjà fait l'objet de cette problématique. La pensée algébrique est notamment sollicitée lors de la conversion du langage naturel au langage algébrique. Ces différents questionnements ont amené les chercheurs à réfléchir sur le lien entre l'arithmétique et l'algèbre. En effet, dans la démarche arithmétique l'inconnue n'est pas opérée. Dans le sujet de l'émergence et de développement conceptuel de l'algèbre, on développe des procédures de résolution de problèmes dans lesquelles l'inconnue est supposée connue (c'est l'aspect analytique) et elle est opérée (c'est l'aspect opération de l'inconnue), (Filloy et Rojano, 1989) [19].

Dans le contexte du système scolaire Marocain, il nous a été utile de dégager des éclairages sur l'activité de l'élève, la nature des objets avec lesquels il résout les problèmes de partage inégaux, les stratégies et les raisonnements qu'il met en œuvre, voire le caractère analytique dans ses raisonnements en lien avec le développement de la pensée algébrique. Nous formalisons notre question par : Quels sont les raisonnements utilisés par les élèves de la 6e primaire et de la première année du collège ? Et Comment peut-on distinguer le raisonnement analytique du raisonnement arithmétique dans la résolution de problèmes se ramenant à la recherche de valeurs inconnues?

Cette étude a porté sur la caractérisation des raisonnements des élèves, nous permet d'expliquer en quoi le degré d'analycité d'un raisonnement est un concept essentiel en apprentissage de l'algèbre pour combler les difficultés observées. Ce qui nous amènes à étudier les procédures de résolution de problèmes chez les élèves avant et après l'entrée à l'algèbre, afin de caractériser les différents types de raisonnements. Pour cette raison, nous supposons que, le développement de la pensée algébrique avant l'introduction de l'algèbre pousse l'élève à produire des raisonnements inventifs. Ce qui nous pousse à proposer aux élèves des problèmes déconnectés, qui favorisent l'émergence du raisonnement analytique, voire les soutiennent à développer la pensée algébrique.

Après avoir présenté la question de recherche, nous pointons une allure théorique de l'enseignement de l'algèbre élémentaire et nous exposerons la distinction entre un raisonnement arithmétique et un raisonnement algébrique. Ensuite nous mettons le point sur la conversion du langage naturel au langage algébrique, en précisant la continuité et la complémentarité entre deux modes de pensée ; tout en survolant à travers un cadre d'analyse 
sur le caractère analytique du raisonnement et la nature du registre de représentation sémiotique. Enfin, nous nous attacherons à faire l'analyse de productions d'élèves concernant les cinq problèmes proposés et dégager les résultats et les enseignements sollicités de l'expérience.

\section{Continuité et rupture entre raisonnement arithmétique et raisonnement algébrique}

L'analyse des raisonnements des élèves débutant en algèbre montre l'existence des discontinuités au niveau du sens attribué au symbolisme en arithmétique et en algèbre, Demonty (2008) [20]. Selon Booth (1984) [14], et Lee et Wheeler (1989) [21], deux éléments se dégagent de ces analyses : le sens que peut prendre le symbole (la lettre), le sens des expressions algébriques et de l'égalité. Sans ignorer qu'il y aura, sans doute, une continuité entre les deux raisonnements arithmétique et algébrique. Parfois, l'arithmétique servira comme outil de vérification des raisonnements algébriques.

Adihou et al (2015) [5] font état de la distinction entre un raisonnement arithmétique et un raisonnement algébrique. Le premier est caractérisé par le fait de partir des données connues et du contexte pour déterminer ce qui sont à chercher. Le second, réside précisément dans le caractère analytique du raisonnement et non dans l'absence ou la présence de lettres pour représenter les inconnues. Adihou et al (2015) [5] font remarquer, comme citée dans les travaux de Mason et Binns (1993) [22], dans une démarche arithmétique, on effectue seulement des calculs sur des quantités connues ; on n'opère pas sur des inconnues. La démarche algébrique consiste à approcher des concepts en jeu dans des activités algébriques, dans une tendance à symboliser et à opérer sur des symboles ou, dans une tendance à avoir une vision structurale des expressions algébriques.

La recherche dans le domaine de la didactique stipule que le rapport entre l'algèbre et l'arithmétique met en jeu une double rupture, la première résulte de l'introduction d'un détour formel dans le traitement de problèmes habituellement traités intuitivement. La seconde, résulte de l'introduction des objets mathématiques nouveaux comme les inconnues, les équations et les variables, Vergnaud (1988) [23]. L'enseignement de l'algèbre avant l'introduction du symbole vise à développer une continuité entre l'arithmétique et l'algèbre par un enrichissement de certains concepts. Pour cela, il faut soutenir le statut d'équivalence du signe d'égalité, induire le caractère structural des expressions arithmétiques et développer la dénotation d'une expression numérique au sens de Pilet (2015) [24].

\section{Conversion du langage naturel au langage algébrique}

La transition de l'arithmétique à l'algèbre exige une discontinuité entre deux styles de pensée mêlant une mutation dans la manière d'expliquer certains concepts mathématiques.

Duval (1991) [25] a avéré que la coordination entre plusieurs registres de représentation sémiotique apparait comme fondamentale pour une appréhension conceptuelle des objets mathématiques, ce qui suppose une discrimination des unités signifiantes des divers registres. La pensée algébrique est notamment sollicitée lors de la conversion du langage naturel au langage algébrique. Cependant, les élèves du secondaire collégial commettent des erreurs d'interprétation, car ils expriment des confusions entre deux formes de langage : le langage naturel et celui algébrique.

L'identification des données pertinentes de l'énoncé d'un problème est une tâche à part entière où l'élève est appelé à être capable de relever la liste des quantités et trouver les regroupements possibles, avant de chercher comment les représenter par une lettre ou un symbole algébrique. Et ensuite, écrire leurs relations et les équations correspondantes. 
L'analyse du passage d'un texte de l'énoncé d'un problème à l'écriture de l'équation algébrique doit donc porter sur deux dimensions essentielles :

- Identifier des quantités inconnues désignées dans l'énoncé et convertir leur expression linguistique en une expression algébrique,

- Identifier des expressions associées aux relations (Didier jean et al, 1997) [26].

Duval (1995) [7] a décrit l'activité mathématique, en analysant sa fonction dans différents langages, comme étant un processus qui fait intervenir trois activités cognitives :

- La formation: Constitution d'une trace dans un système déterminé qui respecte certaines règles de façon à avoir du sens pour celui qui ne va pas lui-même produire.

- Le traitement: Transformation des représentations par des opérations qui s'effectuent dans un même registre.

- La conversion : c'est la transformation d'une représentation en une représentation d'un autre registre.

\section{Cadre d'analyse repérant le degré d'analyticité du raisonnement et la nature du registre de représentation sémiotique}

\subsection{Degré d'analycité du raisonnement}

Nous examinons les résolutions des élèves selon trois grandes catégories de raisonnements. La première regroupe les raisonnements synthétiques, une seconde catégorie regroupe les raisonnements analytiques. La troisième catégorie regroupe les raisonnements dits à tendance analytique qui ne sont pas synthétiques et respectent partiellement les caractéristiques d'un raisonnement analytique (Squalli et al, 2020) [1] :

Raisonnements synthétiques: ce sont des raisonnements qui se caractérisent selon une démarche arithmétique de résolution. Pour déterminer la valeur de l'inconnue, l'élève opère sur des données et des relations connues, il n'opère pas sur l'inconnue.

Raisonnement analytique : Le raisonnement analytique s'explique par la capacité de réfléchir sur les relations et de se servir de l'inconnue comme si elle était connue pour en tirer des conclusions nécessaires.

Raisonnement à tendance analytique : Catégorie de raisonnements hypothético-déductifs, qui ne peuvent pas être classés dans la catégorie des raisonnements de type analytique. Dans cette catégorie de raisonnement, pour rechercher la valeur de l'inconnue, on fait comme si cette valeur existait et on opère sur elle comme on opère sur les nombres connus.

\subsection{Catégorie de raisonnement et nature du registre de représentation sémiotique}

Les registres de représentations sémiotiques indiquent un moyen d'apparence des représentations mentales. Elles sont les résultats dévoilés par le moyen de signes découlant d'un système de représentation (figure, un énoncé en langage courant, formule ou expression algébrique, graphe, schéma), Duval (1991) [25].

- Registre numérique : les traces de la résolution de l'élève ne comportent que les nombres spécifiques et les opérations sur ces nombres.

- $\quad$ Registre algébrique : l'élève recourt au langage algébrique conventionnel.

- Registre intermédiaire: l'élève recourt à un mode de représentations non numériques et non algébriques

Le tableau 1 synthétise les catégories de raisonnements selon le degré d'analycité et la nature du registre de représentation sémiotique : 
Tableau 1. Catégories de raisonnements selon le degré d'analycité et la nature du registre de représentation sémiotique (Squalli et al, 2020) [1].

\begin{tabular}{|l|l|l|}
\hline \multicolumn{1}{|c|}{$\begin{array}{c}\text { Raisonnements } \\
\text { synthétiques }\end{array}$} & \multicolumn{1}{c|}{$\begin{array}{c}\text { Raisonnements à tendance } \\
\text { analytique }\end{array}$} & \multicolumn{1}{c|}{ Raisonnements analytiques } \\
\hline $\begin{array}{l}\text { - Calcul direct ; registre } \\
\text { numérique }\end{array}$ & $\begin{array}{l}\text { - Raisonnement fonctionnel, } \\
\text { - Essais-erreurs, ajustement } \\
\text { rimple; registre numérique intermédiaire (table de } \\
\text { - Essais-erreurs, } \\
\text { Ajustement raisonné }\end{array}$ & $\begin{array}{l}\text { - Type fausse position, } \\
\text { - Inconnues non représentées } \\
\text { explicitement, registre } \\
\text { numérique }\end{array}$ \\
& $\begin{array}{l}\text { - registre intermédiaire } \\
\text { - Inconnue intermédiaire, } \\
\text { registre numérique } \\
\text { algébrique conventionnel mais } \\
\text { sans opération sur les relations } \\
\text { entre les inconnues. }\end{array}$ & $\begin{array}{l}\text { - Registre algébrique } \\
\text { conventionnel, sans perte de lien } \\
\text { aventexte } \\
\text { - Registre algébrique } \\
\text { conventionnel, avec perte de } \\
\text { lien avec le contexte }\end{array}$ \\
& & \\
\hline
\end{tabular}

\section{Méthodologie}

\section{1 Échantillon}

Les participants à cette étude sont des élèves de la 6e année du primaire et du cycle secondaire collégial. Deux niveaux scolaires ont participé à cette étude :

- Des élèves de la 6e année du primaire, à l'âge de (11 à 12 ans).

- Des élèves de la 1ère année du secondaire collégial, leurs âges sont de 12 à 13 ans (Annexe 2, indiquant les niveaux scolaires et tranches d'âges relatifs au système scolaire Marocain).

Nous avons administré un questionnaire formé de cinq problèmes à un échantillon comportant 518 élèves Marocains de 23 classes, qui appartiennent à 19 établissements scolaires du 6e primaire et de la première année du secondaire collégial, en avril-mai 2016 et 2017. Cet échantillon est constitué de 62 élèves du 6e primaire, 456 élèves de la première année du secondaire collégial.

\subsection{Choix des problèmes}

Les énoncés des problèmes, avec leurs structures et les relations de comparaison (Abouhanifa et al, 2018) [27], sont présentés dans le tableau 2 (annexe 1). Ces énoncés soumis aux élèves ont été élaborés de telle sorte que les problèmes se distinguent selon deux sortes de problèmes (Bednarz et Janvier,1996) [28]† :

- Le premier problème présenté est de type connecté, il peut être résolu à partir du nombre de pratiquants du football en exprimant les relations 4 fois et 7 fois.

- Les quatre (de 2 à 5) autres problèmes déconnectés apparaissent comme ceux relevant d'une tendance algébrique. Dans cette classe, les trois types de problèmes se distinguent selon leurs structures, qui ont été prises en compte :

- Source : les deux relations ont la même donnée comme point de départ.

- Composition : une des données est le point d'arrivée d'une relation soit le point de départ de l'autre relation.

\footnotetext{
$\dagger$ Un problème est connecté quand une relation peut facilement être établie entre deux données connues ; donnant la possibilité de raisonner arithmétiquement. Le problème est déconnecté si aucun chemin direct ne peut être établit entre deux données connues.
} 
- Puits : les deux relations ont la même donnée comme point d'arrivée.

Notons que dans chaque structure, trois paramètres ont été pris en compte dans chaque problème, à savoir : Les branches sont en nombre de trois, les relations entre les données sont (additive, multiplicative ou additive et multiplicative) et la spécificité des quantités prises en comptes.

\subsection{Les conditions de passation du questionnaire aux élèves}

Les cinq énoncés de problèmes ont été administrés à chaque élève individuellement. On demandait à chacun d'entre eux de résoudre les cinq problèmes, dont le premier problème avait pour objectif de susciter l'intérêt des élèves et les motiver pour s'engager dans les autres problèmes, en respectant les consignes suivantes :

- $\quad$ Le temps estimé pour répondre au questionnaire est de 50 minutes ;

- Il est interdit d'utiliser la calculatrice ;

- $\quad$ Le travail est individuel ;

- Ne pas effacer, rayer au besoin ;

- Si l'élève ne parvient pas à résoudre un problème, il doit nous expliquer la cause qui l'empêche de l'accomplir.

Afin de collecter plus d'indicateurs sur les différents raisonnements manifestés, nous avons demandé aux élèves de laisser les traces écrites dans la copie, de tous ce qu'ils pensent. Ceci nous a aidés pour avoir un aperçu sur la façon de faire et a permis d'inciter les élèves à réfléchir sur les stratégies utilisées, les tâches et les difficultés rencontrées. Dès que les élèves ont commencé la résolution des problèmes en autonomie, l'enseignant responsable de la classe n'est pas intervenu pendant le processus de résolution, en dehors du fait qu'il a répondu, de façon générale sans donner aucune précision, à quelques questionnements des élèves (les questions posées par les élèves ont porté sur la clarification de l'énoncé du problème, aide demandée pour résoudre les problèmes et la validation de la réponse obtenue).

\subsection{Méthode d'analyse}

En plus des analyses descriptives (effectifs, pourcentages, moyennes) qui nous permettent de donner un aperçu général sur les résolutions des élèves à chacun des problèmes, nous avons recouru, comme l'ont fait d'autres chercheurs (Squalli, Bronner, Larguier et Adihou ; 2020) [1], à une étude qui est fondée sur la grille d'analyse des raisonnements des élèves dans la résolution de problèmes de partages inéquitables. Elle convoite la caractérisation des différents types de raisonnements selon leur degré d'analycité et la nature du registre de représentation sémiotique.

Nous avons considéré qu'un raisonnement est qualifié de raisonnement synthétique, si l'élève trouve la valeur de l'inconnue en n'opérant que sur des nombres ou grandeurs connues. Un raisonnement est qualifié de raisonnement analytique, si l'élève considère l'inconnue, la représente par un symbole et opère sur l'inconnue comme si c'était un nombre connu. Un raisonnement est dit à tendance analytique, si dans ce type de raisonnement qui est ni synthétique ni analytique, l'élève fait comme si la valeur de l'inconnue était connue, mais au lieu d'opérer sur une représentation de cette inconnue, il opère sur une valeur fausse.

Dans cette étude, nous nous attachons particulièrement à documenter les raisonnements qui ne peuvent être qualifiés, ni de raisonnement synthétique explicite, ni de raisonnement algébrique explicite. En effet, les élèves ne maîtrisant pas encore l'outil algébrique, nous nous attendons à ce que la manifestation de tels raisonnements se réalise dans les résolutions des problèmes déconnectés. Nous retenons que le fait d'opérer sur l'inconnue favorise le raisonnement analytique, c'est donc une réflexion sur les relations et les opérations. 


\section{Analyses descriptives des résolutions des élèves selon les catégories de raisonnements}

Les résultats des analyses descriptives résument les résolutions des élèves à travers lesquelles les catégories et les types de raisonnements sont relevés, selon le niveau scolaire et la structure du problème.

\subsection{Les non-réponses}

Le tableau 3 ci-dessous présente le pourcentage de non-réponses (NR) selon le niveau scolaire et le type de problème. Notons que pour un total de 518 copies d'élèves, il nous faudra en somme, un nombre de 2590 résolutions effectives aux cinq problèmes.

Tableau 3. Effectifs et pourcentages de non-réponses à l'un des problèmes au moins.

\begin{tabular}{|l|l|l|l|}
\hline Problème & NR. 6Prim & NR. 1Coll & $\%$ par problème \\
\hline 1 & $4 \%(11)$ & $96 \%(275)$ & $55 \%(286)$ \\
\hline 2 & $12 \%(43)$ & $88 \%(317)$ & $69 \%(360)$ \\
\hline 3 & $16 \%(46)$ & $84 \%(233)$ & $54 \%(279)$ \\
\hline 4 & $13 \%(41)$ & $87 \%(281)$ & $62 \%(322)$ \\
\hline 5 & $8 \%(32)$ & $92 \%(366)$ & $77 \%(398)$ \\
\hline$\%$ NR selon le niveau scolaire & $11 \%(173)$ & $89 \%(1472)$ & $63,51 \%(1645)$ \\
\hline
\end{tabular}

Les données du tableau 3 nous amènes à déduire qu'il y a une majorité de non-réponses au regard du nombre de résolutions de chaque problème, relativement à chaque niveau scolaire. En effet, parmi les 518 copies d'élèves qui sont analysées 63,51\%(1645) d'entre eux ne manifestent aucune réponse à l'un au moins des problèmes proposés, en rapport avec les deux niveaux scolaires promis.

Concernant le niveau 6e primaire, les non-réponses à l'un des problèmes au moins dépassent les $11 \%$ (173). Pour le niveau de la première année collège, 89\% (1 472) des non-réponses se sont manifestées à l'un au moins des problèmes.

Le taux moyen de non-réponses (ou les réponses d'ignorance) en rapport avec tous les niveaux scolaires interrogés atteint $63,51 \%(1645)$. Ce qui peut s'expliquer en grande partie de la méconnaissance des élèves quand il s'agit de résoudre des problèmes de types déconnectés (Abouhanifa et al, 2018) [27]. Le taux de réponses effectives sur l'ensemble des réponses émises par les élèves des deux niveaux scolaires est de 36,49\%(945) réponses à l'un des problèmes au moins. Il rassemble les réponses qui sont réussies et celles non réussies puisqu'elles présentent certaines erreurs. Nous notons que nous n'avions pris en compte dans l'analyse de l'analycité du raisonnement que les erreurs de raisonnement et non celui des calculs.

Nous pouvons signaler aussi, que les réponses d'ignorance des élèves sont incessamment marquées, ceci se manifeste par leurs déclarations à travers les traces écrites « je ne sais pas. » ou par une absence de trace de réponse au problème (la page est restée vide), ce qui est évidemment bien différent. Cela limite les comparaisons possibles dans les taux de réponses d'ignorance d'un problème à l'autre, mais ce type n'empêche pas, en revanche, d'étudier les évolutions des réponses, pour un même problème. Le temps de réponses des élèves était limité, on leur demandait pourtant d'être assez rapides (cinq problèmes en une durée de 50 minutes et les élèves ne sont pas familiarisés avec ce type de problèmes) (ibid). Cette condition expérimentale a pu augmenter le taux de réponses d'ignorance ou de non-réponses. 


\subsection{Distribution des catégories de raisonnements selon le niveau scolaire}

Tableau 4. Répartitions des types de raisonnements selon les niveaux scolaires.

\begin{tabular}{|c|c|c|c|c|c|c|c|c|}
\hline \multirow{2}{*}{ Niveau } & \multicolumn{2}{|c|}{ Synthétique } & \multicolumn{2}{c|}{ Analytique } & \multicolumn{2}{c|}{$\begin{array}{c}\text { A tendance } \\
\text { Analytique }\end{array}$} & \multicolumn{2}{c|}{ Total } \\
\cline { 2 - 9 } & Réussis & Non Réu. & Réussis & Non Réu. & Réussis & $\begin{array}{c}\text { Non } \\
\text { Réu. }\end{array}$ & Réussis & $\begin{array}{c}\text { Non } \\
\text { Réu. }\end{array}$ \\
\hline $\begin{array}{c}6 \mathrm{e} \\
\text { Primaire }\end{array}$ & $\begin{array}{c}41 \% \\
(30)\end{array}$ & $59 \%(43)$ & $27 \%(17)$ & $73 \%(47)$ & $0 \%(0)$ & $0 \%(0)$ & $34 \%(47)$ & $66 \%(90)$ \\
\hline $\begin{array}{c}1 \mathrm{e} \\
\text { Collège }\end{array}$ & $22 \%(90)$ & $78 \%(322)$ & $11 \%(84)$ & $89 \%(268)$ & $25 \%(11)$ & $75 \%(33)$ & $23 \%(185)$ & $\begin{array}{c}77 \%(623 \\
)\end{array}$ \\
\hline Total & $\begin{array}{c}24,74 \% \\
(120)\end{array}$ & $\begin{array}{c}75,26 \% \\
(365)\end{array}$ & $\begin{array}{c}24,28 \% \\
(101)\end{array}$ & $\begin{array}{c}75,72 \% \\
(315)\end{array}$ & $\begin{array}{c}25,00 \% \\
(11)\end{array}$ & $\begin{array}{c}75,00 \% \\
(33)\end{array}$ & $\begin{array}{c}24,55 \% \\
(232)\end{array}$ & $\begin{array}{c}75,45 \% \\
(713)\end{array}$ \\
\hline
\end{tabular}

Les données du tableau 4 mettent en évidence que 24,55\% (232) des raisonnements qui sont réussis, et ceux-ci pour les 232 résolutions effectives à l'un au moins des problèmes et selon les deux niveaux scolaires. Les raisonnements synthétiques (arithmétiques) réussis représentent $24,74 \%$ (120) et $25 \%$ (11) sont des raisonnements à tendance analytiques qui sont réussis et $24,28 \%$ (101) sont des raisonnements analytiques réussis.

Ces informations quantitatives laissent apparaître que le caractère du raisonnement synthétique est dominant par rapport à celui du raisonnement analytique. La catégorie du raisonnement à tendance analytique est bien présente.

Les élèves du primaire interrogés n'ont pas encore reçu un enseignement de résolution de problème par l'algèbre, nous remarquons qu'il y a un taux moyen de $34 \%$ (47) raisonnements réussis à l'un des problèmes (dont $41 \%$ (30) sont des raisonnements synthétiques et $27 \%$ (17) sont des raisonnements analytiques (17), alors que nous n'avons pas distingué de raisonnement à tendance analytique chez cette catégorie d'élèves). Le taux de raisonnements réussis pour les élèves de la première année du collège est de $23 \%$ (185) raisonnements à l'un des problèmes au moins. Le caractère du raisonnement à tendance analytique est dominant par rapport à celui du raisonnement synthétique et analytique. En effet, $25 \%$ (11) sont des raisonnements à tendance analytique qui sont réussis et $11 \%$ (84) sont des raisonnements analytiques qui sont réussis et $22 \%$ (90) sont des raisonnements synthétiques qui sont réussis.

Dans les résolutions des élèves, la présence des raisonnements analytiques et des raisonnements à tendance analytique qui proviennent des deux niveaux scolaires peuvent s'expliquer par le fait qu'il y a des élèves qui ont reçu un enseignement sur la résolution de problème par l'algèbre. Cependant, nous pourrons déduire que, le degré d'analycité dans la résolution se manifeste dans les raisonnements de ces élèves, surtout à partir du niveau $6 \mathrm{e}$ primaire.

Nous notons que la raison d'être du taux de raisonnement synthétique provient en général du premier problème qui est de type connecté. À travers lequel la relation peut facilement être établie entre deux données connues, donnant la possibilité de raisonner arithmétiquement.

\subsection{Catégories de raisonnements par niveau scolaire et selon chaque problème}

\subsubsection{6e année du primaire}

L'échantillon partiel du 6e primaire comportant 62 élèves. En se limitant au nombre de résolutions effectives qui sont en nombre de 137 résolutions, dont $34 \%$ (47) sont des résolutions réussies et $60 \%$ (90) résolutions non réussies, à l'un des problèmes au moins. 
Tableau 5. Catégories de raisonnements des élèves de la 6e primaire pour chaque problème

\begin{tabular}{|c|c|c|c|c|c|c|c|c|}
\hline \multirow{2}{*}{ Problème } & \multicolumn{2}{|c|}{ Synthétique } & \multicolumn{2}{c|}{ Analytique } & \multicolumn{2}{c|}{$\begin{array}{c}\text { À tendance } \\
\text { Analytique }\end{array}$} & \multicolumn{2}{c|}{ Total } \\
\cline { 2 - 9 } & Réussis & $\begin{array}{c}\text { Non } \\
\text { Réu. }\end{array}$ & Réussis & $\begin{array}{c}\text { Non } \\
\text { Réu. }\end{array}$ & Réussis & $\begin{array}{c}\text { Non } \\
\text { Réu. }\end{array}$ & Réussis & Non Réu. \\
\hline 1 & $61 \%(30)$ & $39 \%(19)$ & $40 \%(4)$ & $60 \%(6)$ & 0 & 0 & $58 \%(34)$ & $42 \%(25)$ \\
\hline 2 & $0 \%(0)$ & $100 \%(7)$ & $17 \%(2)$ & $83 \%(10)$ & 0 & 0 & $11 \%(2)$ & $89 \%(17)$ \\
\hline 3 & $0 \%(0)$ & $100 \%(5)$ & $55 \%(6)$ & $45 \%(5)$ & 0 & 0 & $37 \%(6)$ & $63 \%(10)$ \\
\hline 4 & $0 \%(0)$ & $100 \%(6)$ & $20 \%(3)$ & $80 \%(12)$ & 0 & 0 & $14 \%(3)$ & $86 \%(18)$ \\
\hline 5 & $0 \%(0)$ & $100(6)$ & $12 \%(2)$ & $88 \%(14)$ & 0 & 0 & $9 \%(2)$ & $91 \%(20)$ \\
\hline Total & $41 \%(30)$ & $59 \%(43)$ & $27 \%(17)$ & $73 \%(47)$ & 0 & 0 & $34 \%(47)$ & $66 \%(90)$ \\
\hline
\end{tabular}

Le taux de raisonnements synthétiques réussis manifesté par ces élèves à l'un des problèmes au moins est de $41 \%$ (30). Un taux de $27 \%$ (17) raisonnements analytiques réussis à l'un des problèmes au moins a été effectué. Par contre, nous n'avons remarqué aucune production d'une résolution faisant appel à un raisonnement de type à tendance analytique.

Dans ces raisonnements les inconnues et les équations ne sont pas explicitées, mais les élèves opèrent sur les représentations pour trouver l'inconnue. Cela pourrait s'expliquer par le fait que ces élèves n'ont pas encore reçu un enseignement de l'algèbre.

En ce qui concerne la présence des raisonnements analytiques dans les résolutions des élèves de la 6e primaire, nous pouvons déclarer, que même s'ils n'ont pas encore reçu un enseignement de l'algèbre, il y avait un effet d'enseignement de la part de leur enseignant. En effet, ces enseignants ont amené les élèves à exploiter des modèles de représentation à coordonner les registres de représentations sémiotiques pour résoudre des problèmes de type comparaison (un manuel scolaire de la 6e primaire traite un exemple de ce genre).

\subsubsection{1ère année du collège}

Concernant les 456 élèves de la première année du collège interrogés, en se limitant au nombre de résolutions effectives à l'un des problèmes au moins, qui est de 808 résolutions, dont $23 \%$ (185) sont des résolutions réussies et $77 \%$ (623) résolutions non réussies.

Tableau 6. Catégories de raisonnements des élèves de la première année selon chaque problème

\begin{tabular}{|c|c|c|c|c|c|c|c|c|}
\hline \multirow{2}{*}{ Problème } & \multicolumn{2}{|c|}{ Synthétique } & \multicolumn{2}{|c|}{ Analytique } & \multicolumn{2}{c|}{$\begin{array}{c}\text { A tendance } \\
\text { Analytique }\end{array}$} & \multicolumn{2}{c|}{ Total } \\
\cline { 2 - 9 } & Réussis & Non Réu. & Réussis & Non Réu. & Réussis & $\begin{array}{c}\text { Non } \\
\text { Réu. }\end{array}$ & Réussis & Non Réu. \\
\hline 1 & $56 \%(90)$ & $44 \%(71)$ & $33 \%(4)$ & $67 \%(8)$ & $50 \%(4)$ & $50 \%(4)$ & $54 \%(98)$ & $46 \%(83)$ \\
\hline 2 & $0 \%(0)$ & $100 \%(39)$ & $33 \%(30)$ & $67 \%(60)$ & $40 \%(4)$ & $60 \%(6)$ & $24 \%(34)$ & $76 \%(105)$ \\
\hline 3 & $0 \%(0)$ & $100 \%(41)$ & $25 \%(40)$ & $75 \%(122)$ & $10 \%(2)$ & $90 \%(18)$ & $19 \%(42)$ & $81 \%(181)$ \\
\hline 4 & $0 \%(0)$ & $100 \%(91)$ & $13 \%(10)$ & $87 \%(68)$ & $17 \%(1)$ & $83 \%(5)$ & $6 \%(11)$ & $94 \%(164)$ \\
\hline 5 & $0 \%(0)$ & $100 \%(80)$ & $0 \%(0)$ & $100 \%(10)$ & $0 \%(0)$ & $0 \%(0)$ & $0 \%(0)$ & $100 \%(90)$ \\
\hline Tot & $22 \%(90)$ & $78 \%(322)$ & $11 \%(84)$ & $89 \%(268)$ & $25 \%(11)$ & $75 \%(33)$ & $23 \%(185)$ & $77 \%(623)$ \\
\hline
\end{tabular}


Pour les cinq problèmes proposés, le taux de raisonnements synthétiques réussis manifesté par ces élèves est de $22 \%(90)$ résolutions par rapport à $78 \%(322)$ résolutions non réussies. Un taux de $11 \%(84)$ raisonnements analytiques réussis à l'un des problèmes au moins par rapport à $89 \%(268)$ résolutions non réussies. Quant au raisonnement de type à tendance analytique, nous avons remarqué qu'il y a $25 \%$ (11) résolutions réussies et $75 \%$ (33) résolutions non réussies. Nous notons ici que les élèves de la première année du collège sont initiés à une entrée de l'enseignement de l'algèbre et il nous semble que le recours au raisonnement analytique est conforme à ce qui est attendu du programme, mais nous pensons que le recours au raisonnement de type à tendance analytique dans les résolutions, à ce niveau scolaire et à la période du test, peut-être expliqué par le fait que ces élèves n'ont pas encore bénéficié de la maîtrise nécessaire du sens de la lettre et d'opérer sur les inconnues et leurs représentations.

\section{Catégories de raisonnements et registres de représentations sémiotiques illustrées d'exemples de productions des élèves}

\subsection{Raisonnement de type synthétique}

\subsubsection{Raisonnement basé sur un calcul direct}

Cette famille de raisonnements regroupe les raisonnements arithmétiques coutumiers dans le cas de problèmes connectés. À travers un raisonnement basé sur un calcul direct, l'élève opère sur les données et les relations connues pour trouver la valeur de l'inconnue.

\begin{tabular}{|c|c|}
\hline 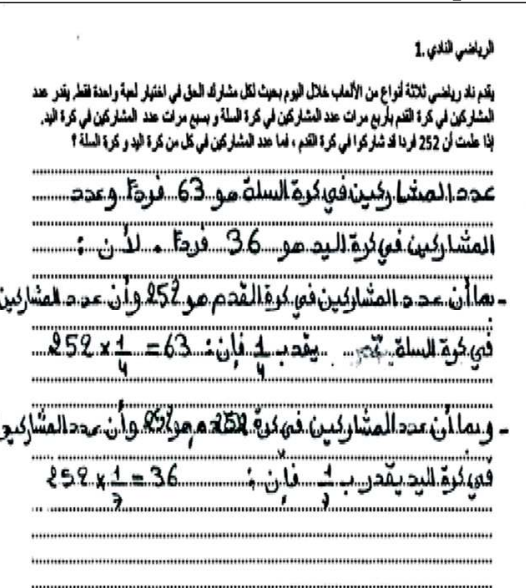 & 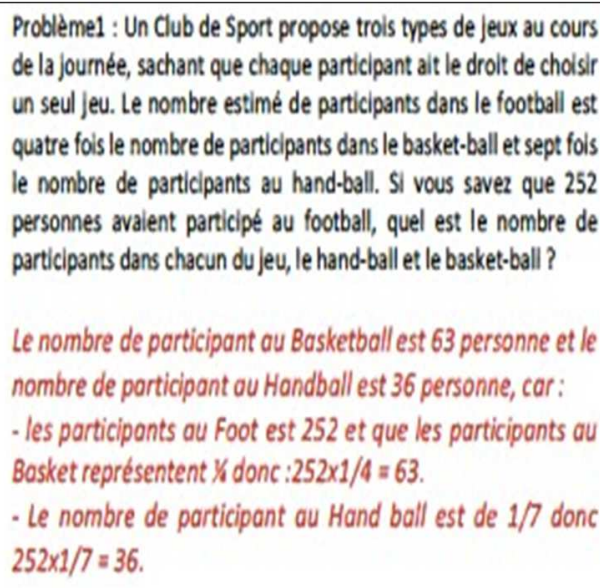 \\
\hline
\end{tabular}

Fig. 1. Production de l'élève 6P9 (avec la traduction libre).

L'élève commence par représenter les relations telles qu'elles apparaissent dans l'énoncé du problème. Il exprime ces relations par $1 / 4$ du nombre de pratiquants du football qui est 252 et $1 / 7$ du nombre de pratiquants du football qui est 252 , exprimant le nombre de pratiquant du basket-ball et d'handball, respectivement. Le problème est un problème connecté de type puits. L'élève 6P9 (Figure 1) a bien compris que ce problème dont la nature des relations entre les quantités est multiplicative. Il calcule les valeurs des deux inconnues, le nombre de participant au Basket-Ball et le nombre de participant au Handball en utilisant les relations connues entre ces deux inconnues et la quantité connue - le nombre de participants au Football (252). Il déduit alors le total des participants au Basket-Ball et au handball. 
Le registre de représentation sémiotique utilisé est le registre numérique vu que les traces de la résolution de l'élève ne comportent que les nombres spécifiques et les opérations sur ces nombres.

\subsection{Raisonnement analytique}

\subsubsection{Inconnues non représentées explicitement, registre numérique}

Dans cette catégorie du raisonnement analytique, l'inconnue et l'équation sont muettes bien qu'elles soient objets de la pensée de l'élève. Alors que le registre est uniquement numérique. Exemple 1

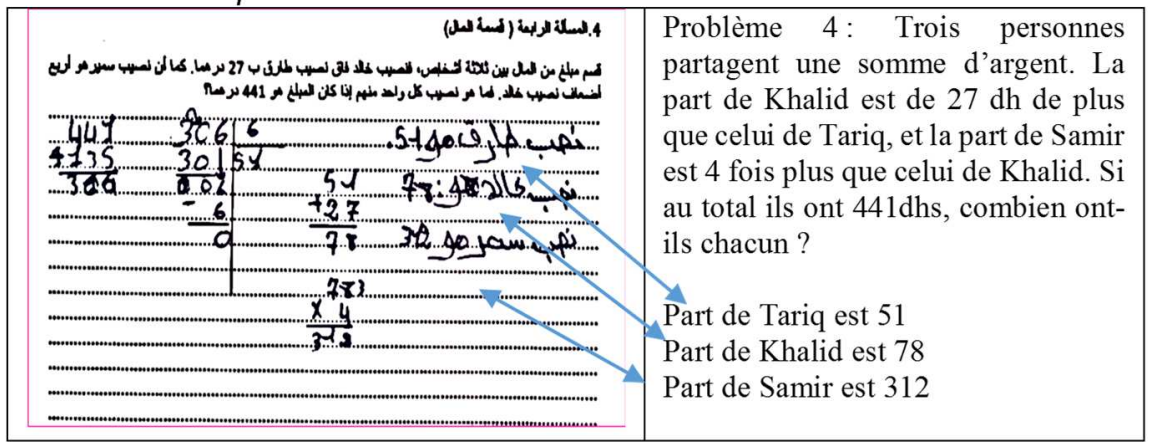

Fig. 2. Production de l'élève 6P25 (avec la traduction libre).

L'élève 6P25 (Figure 2) entame sa résolution du problème 4 en mobilisant les données et les relations connues pour trouver la valeur de l'inconnue comme dans le cas d'une démarche de résolution algébrique conventionnelle. Il soustrait une valeur 135 du total de 441 ; (441 - 135 $=306)$. Il divise le résultat trouvé par $6 ;(306: 6=51)$ et il trouve la part de Tariq.

La multiplication de 27 par 5 et la soustraction de 135 du total de 441 ainsi que la division du résultat trouvé 306 par 6 ; ils montrent que l'élève saisit bien l'équation du problème. Cette procédure découle d'une bonne interprétation des relations entre les trois inconnues : si la part de Khalid est de 27 dhs de plus que celui de Tariq et que la part de Samir est 4 fois plus que celui de Khalid, alors 6 fois la part de Tariq est égale au total de 441 auquel on retranche $(27+4 \times 27=135)$.

\section{Exemple 2}

\begin{tabular}{|c|c|}
\hline 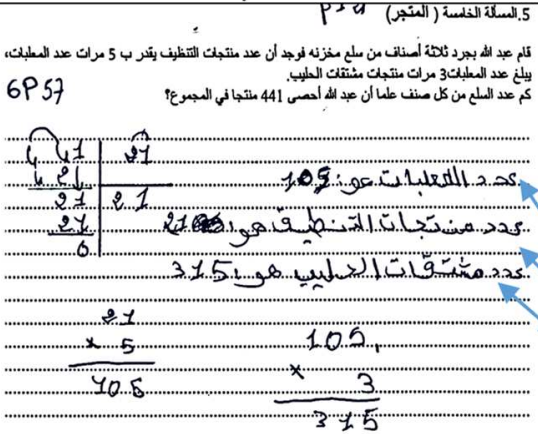 & $\begin{array}{l}\text { Problème } 5 \text { : Abdellah fait l'inventaire de trois } \\
\text { produits de sa boutique. Il compte } 5 \text { fois plus } \\
\text { de produites de conserve que de produits } \\
\text { nettoyage et il compte } 3 \text { fois plus de produits } \\
\text { laitiers que de produits en conserve. S'il y a } \\
441 \text { produits en tout, combien y a-t-il de } \\
\text { produits de chaque type? } \\
\text { Le nombre de produits de conserve est : } 105 \\
\text { Le nombre de produits en nettoyage est : } 21 \text {. } \\
\text { Le nombre de produits laitiers est }: 315\end{array}$ \\
\hline
\end{tabular}

Fig. 3. Production de l'élève 6P57 (avec la traduction libre)

Le registre de représentation est uniquement numérique. Dans ce raisonnement, l'inconnue principale, qui est la part de Tariq, n'est pas représentée explicitement ni l'équation mathématisant le problème. Donc, l'inconnue et l'équation sont muettes bien qu'elles soient objets de la réflexion de l'élève. C'est un raisonnement analytique basé sur le calcul dans le 
registre numérique. Le registre est numérique, mais il ne rend pas compte de tout le raisonnement de l'élève. Car, il est difficile d'affirmer que cet élève a opéré sur l'inconnue. Le même raisonnement manifesté par l'élève $6 \mathrm{P} 25$ pour résoudre le problème 4 (Figure 2) est employé par l'élève 6P57 pour résoudre le problème 5 (Figure 3). En effet, à travers des calculs numériques posés, l'élève divise 441 par 21, il trouve la valeur 21 qu'il accorde au nombre de produits en nettoyage. Ensuite, il multiplie ce nombre 21 par 5 et puis il multiplie la valeur trouvée par 3 pour trouver respectivement, 105 le nombre de produits de conserve et 315 le nombre de produits laitiers.

La division par 21 montre que l'élève saisit bien l'équation du problème. Cette procédure découle d'une bonne interprétation des relations entre les trois inconnues : s'il compte 5 fois plus de produits de conserve que de produits nettoyage et il compte 3 fois plus de produits laitiers que de produits en conserve, alors ( 5 fois +15 fois +1 fois) le nombre de nettoyage est égal au total de 441 . Le registre de représentation est uniquement numérique. Dans ce raisonnement, l'inconnue principale, qui est le nombre de produits nettoyage, n'est pas représentée explicitement ni l'équation mathématisant le problème. Donc, l'inconnue et l'équation sont muettes bien qu'elles soient objets de la pensée de l'élève. C'est un raisonnement analytique alors que le registre de représentation est exclusivement numérique.

\subsubsection{Inconnue intermédiaire, registre numérique}

Dans cette catégorie du raisonnement analytique, l'élève opère sur une inconnue intermédiaire. Cette inconnue et l'équation correspondante sont muettes bien qu'elles soient objets de la pensée de l'élève. Alors que le registre est uniquement numérique.

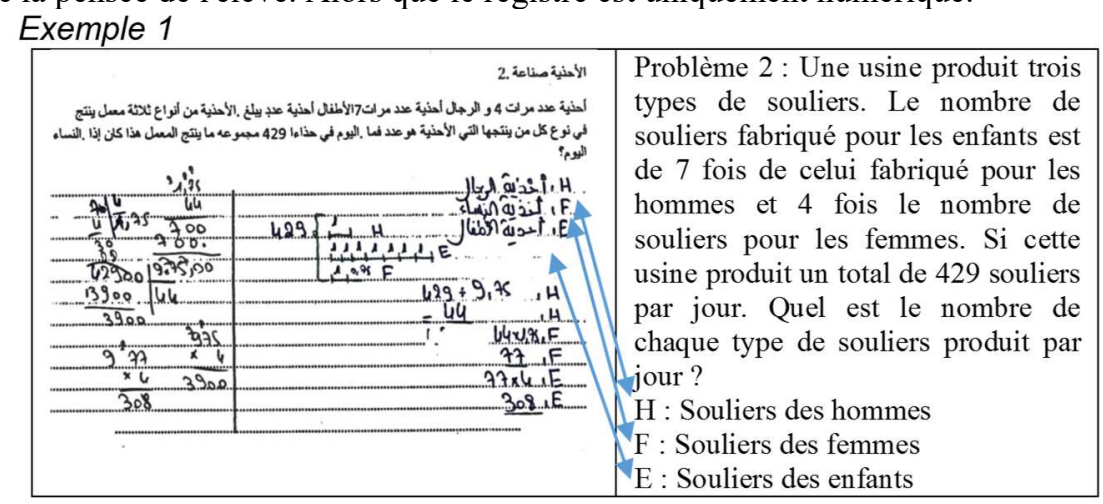

Fig. 4. Production de l'élève 6P11 (avec la traduction libre).

L'élève 6P11 (figure 4) semble saisir la structure multiplicative du problème 2. Il a représenté le nombre de souliers des hommes par « une partie », le nombre de souliers des enfants est 7 fois, donc « 7 parties » et le nombre de souliers des enfants est 4 fois celui des femmes, donc « 7/4 parties » c'est-à-dire une partie à laquelle il ajoute 0,75 . Le total des nombres de souliers des trois personnes étant le tout, c'est-à-dire 429. Le terme "partie » est un substitut de l'inconnue, il joue le rôle d'inconnue intermédiaire dont il faut calculer la valeur en nombre de souliers. Le tout est donc constitué de « 9,75 parties » qui totalisent 429 souliers.

Pour trouver la valeur d'une partie en nombre de souliers, l'élève commence par une division de 429 par 9,75, il trouve la valeur 44, qui représente le nombre de souliers des hommes. Par une multiplication de 44 par 1,75, il en déduit que le nombre de souliers des femmes est de 77. Il en déduit ensuite, le nombre de souliers des enfants 308, en effectuant une multiplication de 44 par 7. L'inconnue et l'équation sont muettes bien qu'elles soient objets de la pensée de l'élève. 
Le registre est numérique, car les traces de la résolution de l'élève ne comportent que des nombres spécifiques et des opérations sur ces nombres. Il a utilisé aussi, le registre des schémas qui est traité comme des parties « longueur du segment ». Cependant, le registre du langage naturel est considéré comme trace du raisonnement de l'élève qui a introduit cette inconnue intermédiaire. Notons toutefois que pour représenter les relations l'élève a eu recours à représenter l'inconnue de référence et les relations comme mesure de longueurs.

Dans la résolution du problème 3, nous notons tout d'abord que pour représenter les relations, l'élève 6 P23 a eu recours à la représentation de l'inconnue de référence et les relations comme mesure de longueurs «partie ». Le terme «partie » est un substitut de l'inconnue, il joue le rôle d'inconnue intermédiaire dont il faut calculer sa valeur en dh. Pour trouver la valeur d'une « partie » représentée par alimentation, une partie plus 850 pour habillement et une partie plus 870 pour enseignement, l'élève commence par soustraire de la somme totale 4960 la somme $850+870$ ainsi qu'il effectue une division du résultat trouvé 3240 par 3 . Il en déduit les frais d'alimentation 1080dhs. Ensuite, il ajoute 850 à 1080 pour trouver les frais d'habillement et il ajoute 870 à 1080 pour trouver les frais d'enseignement. Cet élève n'utilise pas de représentation explicite des inconnues, des relations et de l'équation, mais son traitement numérique montre qu'il opère sur les inconnues et non sur les relations. L'élève a produit un raisonnement analytique l'inconnue et l'équation sont muettes. Le registre du langage naturel est considéré comme trace du raisonnement de l'élève qui a introduit cette inconnue intermédiaire.

\subsubsection{Inconnue intermédiaire, registre intermédiaire}

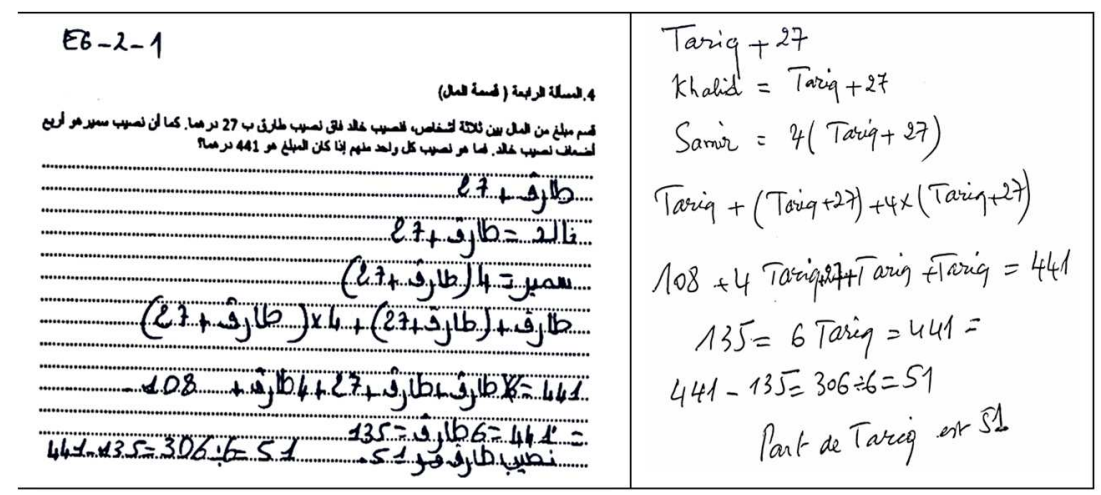

Fig. 7. Production de l'élève 6P1 (avec la traduction libre)

L'élève 6P1 (figure 7) semble saisir la structure du problème 4 avec les relations additive et multiplicative. Le total à partager entre les trois personnes étant le tout, Tariq est en une partie, Khalid en une autre partie (celle de Tariq auquel en ajoute 27) et Samir en a 4 fois plus que la part de Khalid, donc 6 parties (celle de Tariq) auxquelles en ajoute 5 fois 27, le tout totalise $441 \mathrm{dhs}$. Le terme «Tariq» est un substitut de l'inconnue, il joue le rôle d'inconnue intermédiaire dont il faut calculer sa valeur en dh. Pour trouver la valeur d'une partie de «Tariq » en dh, l'élève commence par une multiplication de 27 par 5 et la soustraction de 135 du total de 441 ainsi que la division du résultat trouvé 306 par 3. Il en déduit la part de Tariq $51 \mathrm{dh}$. L'inconnue et l'équation sont muettes bien qu'elles soient objets de la pensée de l'élève.

Le registre est intermédiaire, car l'élève recourt à un mode de représentations non numériques et non algébriques. Cependant le registre du langage naturel est considéré comme trace du raisonnement de l'élève qui a introduit cette inconnue intermédiaire. 


\subsubsection{Inconnues explicites, registre intermédiaire}

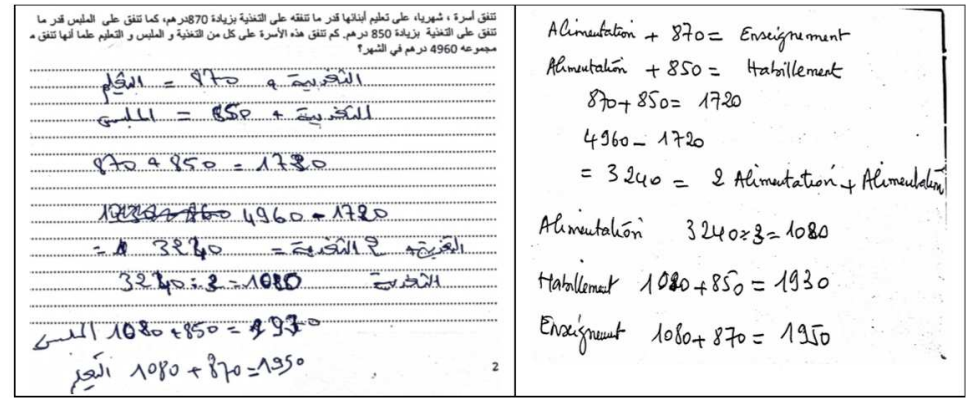

Fig. 8. Production de l'élève 1C89 (avec la traduction libre).

L'élève 1C89 (figure 8) traduit les trois relations entre les inconnues en représentant les inconnues par les noms des objets ; « Enseignement =Alimentation+870 » et « Habillement $=$ Alimentation $+850 »$. Son calcul $870+850=1720$ et 4960-1720 = 3240 montre qu'il a additionné les écarts des inconnues «Enseignement» et «Habillement » à l'inconnue «Alimentation », puis déduit le résultat du montant total des trois inconnues (4 960). Ensuite, il a utilisé la troisième relation « implicitement » qui stipule que la somme totale des frais est égale à 4960 . Cela lui permet de trouver l'équation modélisant le problème en substituant les inconnues «Habillement» et «Enseignement» par leurs relations avec l'inconnue principale "Alimentation» et en tenant compte du calcul précédent: Alimentation+ 2Alimentation $=3240$. En divisant 3240 par 3, il obtient les frais de l'Alimentation est 1080dh. Il déduit alors les valeurs des deux autres inconnues en réalisant les calculs numériques et en faisant référence au contexte du problème.

Ce raisonnement est donc analytique, avec un registre intermédiaire. En effet, l'élève a bien considéré les inconnues, les a représentées explicitement et a opéré sur ces représentations pour former l'équation et trouver les valeurs des inconnues.

\subsubsection{Inconnue intermédiaire, registre intermédiaire en faisant référence au contexte du problème}

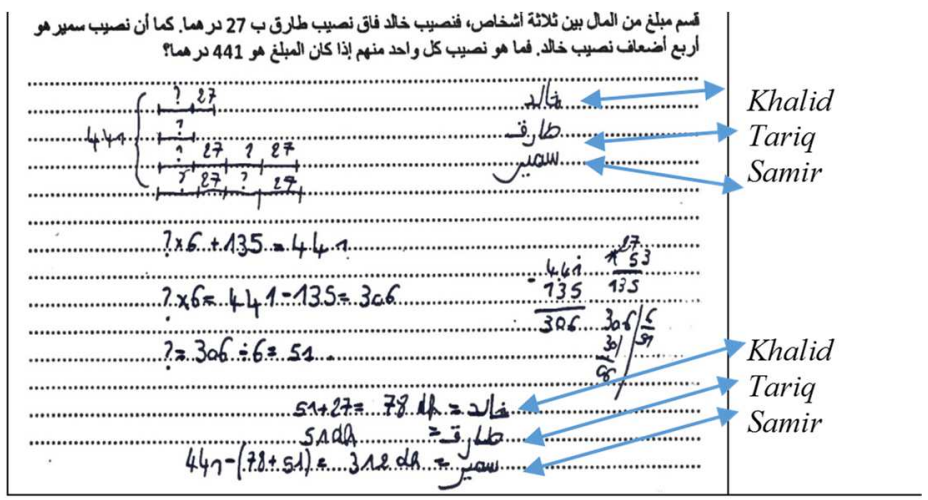

Fig. 9. Production de l'élève 6P9 (avec la traduction libre).

L'élève 6P9 (figure 9) entreprend sa résolution selon une démarche qui respecte les caractéristiques de la résolution algébrique. Il traduit les relations dans le problème en représentant les quantités inconnues par les noms des personnes. Il exprime l'inconnue «?» ayant la plus petite valeur (part de Tariq). Il exprime ensuite l'équation mathématisant le problème en fonction de l'inconnue «? ». Il compte le nombre d'occurrences de l'inconnue 
«?» et la valeur totale des deux nombres dans l'équation. Puis il réalise les calculs numériques pour trouver la valeur de l'inconnue «? » en faisant référence au contexte du problème.

Ce raisonnement est donc analytique, avec un registre intermédiaire. En effet, l'élève a bien considéré l'inconnue «? ", représentant la part de Tariq de façon explicite, et il a opéré sur ces représentations pour former l'équation et trouver les valeurs des inconnues.

\subsubsection{Registre de représentation algébrique, sans perte de lien avec le contexte}

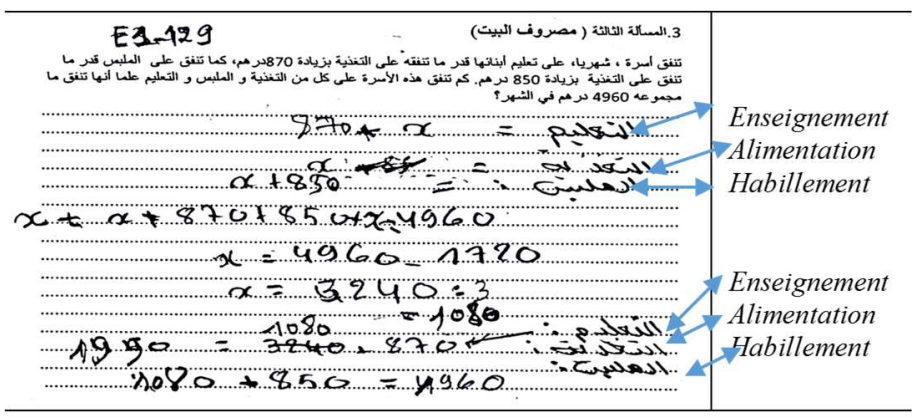

Fig. 10. Production de l'élève 1C129 (avec la traduction libre).

L'élève entreprend sa résolution selon une démarche de résolution algébrique. Il traduit les relations dans le problème en représentant l'inconnue «Alimentation » par x. Il exprime les deux autres inconnues « Enseignement » par $x+850$ et « Habillement » par $x+870$. Il exprime ensuite l'équation mathématisant le problème en fonction de l'inconnue $x$. L'élève utilise donc de façon correcte le registre algébrique pour former les équations, mais il ne sait pas utiliser les règles de traitement et pour cela il a recours au registre numérique. Il réalise les calculs numériques pour trouver la valeur de l'inconnue comme dans le cas des raisonnements à tendance analytique où l'inconnue est muette. Son raisonnement reste attaché au contexte du problème. Le fait d'avoir choisi l'inconnue $\mathrm{x}$ « Alimentation » comme inconnue de référence, dont la valeur est la plus petite des trois, fait en sorte que tous les calculs réalisés ont une signification dans le contexte.

\subsubsection{Registre de représentation algébrique avec perte de lien avec le contexte}

\begin{tabular}{|c|c|}
\hline 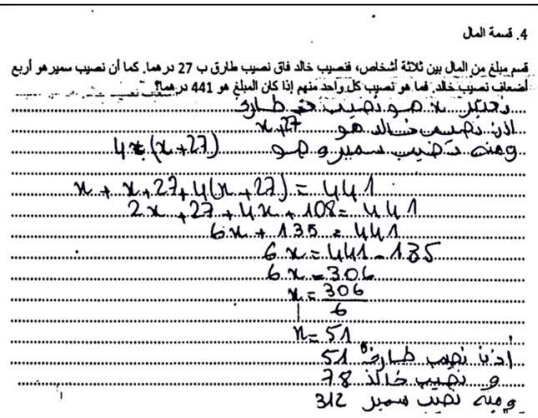 & $\begin{array}{l}\text { Probline 4: } \\
\text { On considere } x \text { la part de Tariq. } \\
\text { Done la part de khalid est : } x+27 \\
\text { it la part de Samir est } 4 \times(x+27) \\
x+x+27+4(x+27)=441 \\
2 x+27+4 x+108=441 \\
6 x+135=441 \\
6 x=441-135 \\
6 x=306 \\
x=\frac{306}{6} \\
x=51 \\
\text { Donc la part de Tarig est.51 } \\
\text { la part de Kalid } 78 \\
\text { ls part de Samir } 312\end{array}$ \\
\hline
\end{tabular}

Fig. 11. Production de l'élève 1C259 (avec la traduction libre).

La résolution de l'élève 1C259 illustre une démarche algébrique. Par une action de traitement, l'élève a opéré sur l'équation pour la simplifier et isoler l'inconnue $\mathrm{x}$ en utilisant 
les transformations algébriques sans rattachement au contexte du problème. Le raisonnement est analytique et le registre de représentation algébrique avec perte de lien avec le contexte.

\subsection{Raisonnement à tendance analytique}

\subsubsection{Raisonnement de type fausse position, registre numérique}

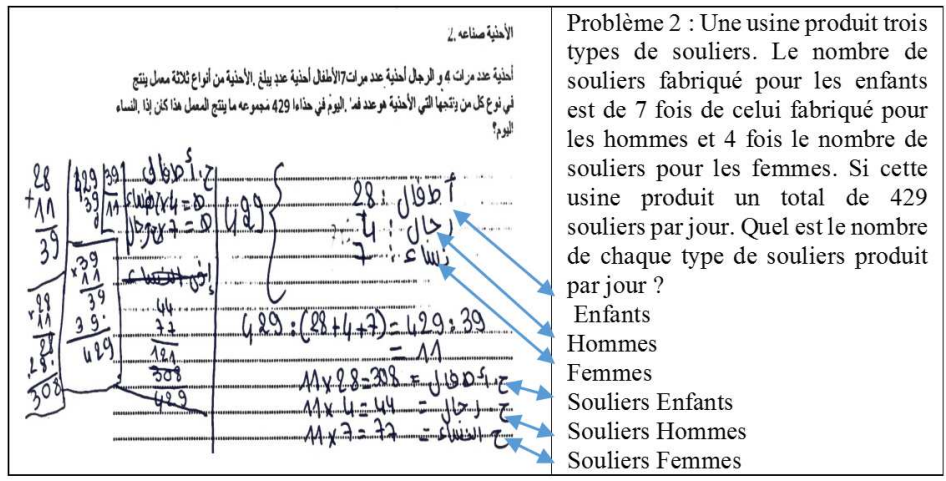

Fig. 12. Production de l'élève 1C319 (avec la traduction libre).

L'élève 1C319 (figure 12) identifie et nomme les trois inconnues par des mots (souliers enfants, hommes et femmes). Il a traduit les relations en fonction du nombre de souliers des enfants $(E)$; il a noté : $E=$ le nombre de souliers des hommes qu'on multiplie 7 et $E=$ le nombre de souliers des femmes qu'on multiplie par 4 . Ensuite, il affecte la valeur 7 au nombre de souliers des hommes, 4 au nombre de souliers des femmes et le produit $7 \mathrm{x} 4=28$ à celui des enfants. Il calcule le total des trois valeurs et obtient 39. Il divise le total 429 par cette valeur 39 il trouve 11 . Il multiplie chacune de ces valeurs par 11 pour trouver les valeurs des trois inconnues dont la somme donne le total des souliers 429 .

\subsubsection{Inconnues explicites, registre algébrique conventionnel}

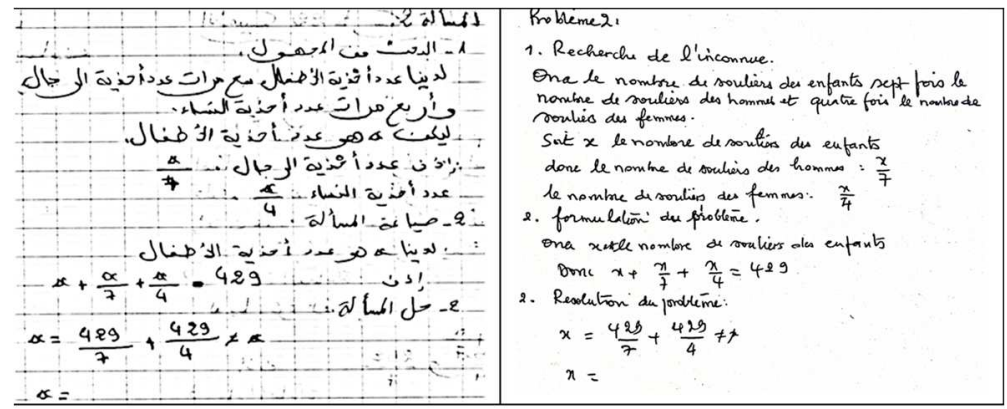

Fig. 13. Production de l'élève 1C409(avec la traduction libre)

En affectant les valeurs 4, 7 et 28, respectivement aux inconnues, nombre de souliers des hommes, nombre de souliers des femmes et nombre de souliers des enfants ; l'élève fait comme si ces inconnues possédaient ces valeurs, il sait manifestement que ces valeurs sont fausses, la suite de sa résolution montre qu'il sait comment corriger ces valeurs initiales après avoir obtenu le total de la somme des trois inconnues. Nous sommes donc bien dans le 
scénario des raisonnements de type fausse position. Ce raisonnement est de type hypothéticodéductif, c'est un raisonnement à tendance analytique alors que le registre de représentation est exclusivement numérique.

L'élève 1C409 (figure 13) entreprend sa résolution selon une démarche de résolution algébrique. Il traduit les relations dans le problème en représentant l'inconnue « nombre de souliers des enfants » par x. Il exprime les deux autres quantités inconnues « le nombre de souliers des femmes » par $\mathrm{x} / 4$ et « le nombre de souliers des hommes» par $\mathrm{x} / 7$. Il exprime correctement l'équation mathématisant le problème en fonction de l'inconnue $\mathrm{x}$. L'élève représente les inconnues par des lettres, utilise ces lettres pour représenter les relations et l'équation mais n'opère pas sur ces représentations. Il utilise donc de façon correcte le registre algébrique pour former les équations, mais il ne sait pas utiliser les règles de traitement et pour cela, il a recours au registre numérique et il abandonne la résolution par la suite. C'est un raisonnement à tendance analytique où l'inconnue est explicite et le registre est algébrique conventionnel.

\section{Conclusion}

L'étude portée au Maroc tend à montrer ainsi, comme dans le cas de l'enquête québécoise (Adihou, Squalli, Saboya, Tremblay, M. Lapointe, A., 2015) [5] et (Squalli, Larguier, Bronner, et Adihou, 2020) [29], qu'en proposant aux élèves des problèmes de type déconnecté (Bednarz, N. et Dufour-Janvier, B., 1992) [30], avant l'enseignement de la méthode algébrique conventionnelle de résolution de problèmes, cela pousse certains élèves à produire des raisonnements qui sont analytiques et des raisonnements qui ne sont ni synthétiques, ni analytiques. Nous avons enregistré aussi que les raisonnements de type essais erreurs peuvent être utilisés pour résoudre les problèmes déconnectés sans recours au raisonnement analytique, ils sont plus fréquents dans les recherches menées en France et au Canada, mais ils ne sont pas abordés dans les productions des élèves marocains de notre échantillon.

La résolution de problèmes déconnectés a permis aux élèves de mettre en évidence des stratégies leur permettant de produire des raisonnements sophistiqués. La structure du problème manifestée par les liens établis entre les relations et le nombre de branches, influence le processus de la résolution

L'analyse montre qu'il y a des apprentissages à explorer chez les élèves dans le passage de l'arithmétique à l'algèbre, qui comportent des traces d'analycité. Les raisonnements manifestés dépasseront celles de la pensée arithmétique qui tendent vers des raisonnements analytiques, où l'inconnue et l'équation ne sont pas explicites. Même si les élèves de la 6e primaire n'ont pas encore été introduits à l'algèbre, les catégories de raisonnement analytique et à tendance analytique, bien que peu représentées, sont également présentes, mais essentiellement chez les élèves des classes de la première année du collège.

Il nous semble alors judicieux d'initier les élèves dès le primaire à ces raisonnements pour les amener à une réflexion sur les caractéristiques de la pensée algébrique avant l'introduction de l'algèbre, et d'étudier les problèmes qui ont du potentiel pour développer des raisonnements analytiques. Voire, les amener à exploiter des modèles de représentations et coordonner entre les registres de représentations sémiotiques.

\section{Références}

1. H. Squalli, M.Larguier, A. Bronner et A. Adihou. Cadre d'analyse des raisonnements dans la résolution de problèmes algébriques de type partage inéquitable. Dans (Squalli, 
H. et Bronner, A. rédacteurs invités). Le développement de la pensée algébrique avant l'introduction du langage algébrique conventionnel. NCRÉ (2020).

2. P. Marchand et N. Bednarz. L'enseignement de l'algèbre au secondaire : une analyse des problèmes présentés aux élèves. Bulletin de l'Association Mathématique du Québec, XXXIX (4), 30- 42(1999).

3. P. Marchand et N. Bednarz. Développement de l'algèbre dans un contexte de résolution de problèmes : résolution des élèves. Bulletin de l'Association des Mathématiques du Québec, XL (4), 15-24 (2000).

4. M. Saboya, V. Besançon, F. Martin, A. Adihou, H.Squalli et M. Tremblay. Résolution de problèmes écrits au moment de l'introduction de l'algèbre : analyse de productions d'élèves du premier cycle du secondaire. Actes du colloque du Groupe des didacticiens des mathématiques du Québec 2013, 112-122 (2014).

5. A. Adihou, H. Squalli, M. Saboya, M. Tremblay et A. Lapointe. Analyse des raisonnements d'élèves à travers des résolutions de problèmes de comparaison, Actes EMF 2015 - GT3 (2015).

6. P. Marchand. Résolution de problèmes en algèbre au secondaire : analyse de deux approches et des raisonnements des élèves. Mémoire de maîtrise en mathématiques, option enseignement, Université du Québec à Montréal (1998).

7. R. Duval. Sémiosis et pensée humaine, Registres sémiotiques et apprentissages intellectuels, Peter Lang SA (1995).

8. L. Lee. La compréhension algébrique : la recherche d'un modèle dans la communauté d'éducation mathématique ; thèse de doctorat, Montréal : université Québec à Montréal (1997).

9. J. Kaput. A research base supporting long term algebra reform. In D. T. Owens. M., K. Reed et G.M. Millaps (dir.) Proceeding of the seventeeth ammial meeting. North american chapter of the international group for the psychology of mathématical education (voli.1, p.71-93). Columbus, OH: the ohio state university (1995).

10. L. Radford. Algebraic Thinking and the Generalization of Patterns: A Semiotic Perspective. In S. Alatorre, J. L. Cortina, M. Sáiz, A. Méndez (Eds.), Proceedings of the 28th Conference of the International Group for the Psychology of Mathematics Education, North American Chapter, Mérida: Universidad Pedagógica Nacional, November 9-12, Vol. 1, p. 2-21 (2006).

11. L. Radford. Iconicity and Contraction: A Semiotic Investigation of Forms of Algebraic Generalizations of Patterns In Different Contexts. ZDM - The International Journal on Mathematics Education. DOI 10.1007/s11858-007-0061-0 (2008).

12. L.Radford. The progressive development of early embodied algebraic thinking. Mathematics Education Research Journal, 26, 257-277 (2014).

13. A. Sierpinska. Interaction des perspectives épistémologique, cognitive et didactique. Dans G. Lemoyne ; F. Conne(dir.), Le cognitifen didactique des mathématiques. Montréal: Presses de l’Université de Montréal, (pp. 151-176) (1999).

14. L. Boot. Algebra: Children's strategies and errors. Windsor, UK: Nfer- Nelson (1984).

15. Y. Chevallard. Le passage de l'arithmétique à l'algébrique dans l'enseignement des mathématiques au collège. Deuxième partie. Perspectives curriculaires : la notion de modélisation. Petit x, 19, 43-72(1989).

16. C. Kieran A functional approach to the introduction of algebra: some pros and cons, in Ponte J. P., Matos J. F. (Eds.). Proceedings of the 18th International Conference for the Psychology of Mathematics Education, 1, 157-175(1994). 
17. J. Vlassis, et I. Demonty. Les représentations pré-algébriques des élèves sortant de l'enseignement primaire. Informations Pédagogiques vol. 47, (1999).

18. K. Subramaniam. Teaching arithmetic and algebraic expressions. Proceedings of the 28e Conference of the International Group for the Psychology of Mathematics Education, vol. 3 (p. 121-128). Mumbai, Inde (2004).

19. E. Filloy et T. Rojano. Solving Equations: The transition from Arithmetic to Algebra" For the learning of mathematics, 9 (2), p. 19-25 (1989).

20. I. Demonty. La transition entre l'arithmétique et l'algèbre élémentaire dans le contexte de la résolution de problèmes arithmétiques. Chapitre 9 In, Fayol, M. (2008). Enseignement et apprentissage des mathématiques. De Boeck, Bruxelles (2008).

21. L. Lee et D. Wheeler. The arithmetic connection. Educational Studies in Mathematic, 20, pp 41-54(1989).

22. J. Mason et L.Binns. Exploration of Vergnaud's theorem-in-action in the context of algebra. A contribution at the ESRC Seminar Group on Algebraic processes and the Role of symbolism. London: University of London (1993).

23. G. Vergnaud, A. Cortes et P. Favre-Artigue. Introduction de l'algèbre auprès de débutants faibles. Problèmes épistémologiques et didactiques. In Vergnaud, G., Brousseau, G., Hulin, M. (Eds.), Didactique et acquisition des connaissances scientifiques. (pp. 259-279). Grenoble : La Pensée Sauvage (1988).

24. J. Pilet. Réguler l'enseignement en algèbre élémentaire par des parcours d'enseignement différencié. Recherches en Didactique des Mathématiques, 35(3), 273-312 (2015).

25. R. Duval. Registres de représentation sémiotique et fonctionnement cognitif de la pensée, Annales de didactique et de sciences cognitives, 5, 37-65 (1991).

26. G.Didierjean, C.Dupuis, R. DUVAL, M-A.Egret, D.Kremer, G.Robert, B.Wenner, M. Ziegler. A propos de charades dont la solution est un système d'équations à deux inconnues. Petit $x$, N 44, pp. 35- 48. IREM de Strasbourg (1997).

27. S. Abouhanifa, H. Squalli, B. Seddoug, et M. El ibbaoui,. Les défauts de représentations sémiotiques à la genèse des difficultés de mise en équation chez des élèves de $2 \mathrm{e}$ et $3 \mathrm{e}$ années du collège. RMDM. Volume 3. (http://geo-top.org/RMDM/index.php/volume3/) (2018).

28. N. Bednarz et B. Dufour-Janvier. Emergence and development of Algebra as a problem solving tool: continuities and discontinuities with arithmetic. In N. Bednarz, C. Kieran et L. Lee (Eds.), Approaches to algebra: perspectives for research and teaching (pp. 115136). Dordrecht: Kluwer (1996).

29. H. Squalli, M. Larguier, A. Bronner \& A. Adihou. Cadre d'analyse des raisonnements dans la résolution de problèmes algébriques de type partage inéquitable. Nouveaux cahiers de la recherche en éducation, 22 (1), 36-62. https://doi.org/10.7202/1070024ar(2020).

N. Bednarz et B. Dufour-Janvier. L'enseignement de l'algèbre au secondaire : une caractérisation du scénario actuel et des problèmes qu'il pose aux élèves. Actes du colloque international du 20 au 22 mai 1992 : didactique des mathématiques, formation normale des enseignants. École normale supérieur Marrakech. p. 21-40 (1992). 
Annexe1 : Tableau 2 : Enoncés des problèmes avec leurs structures et les relations de comparaison (Abouhanifa et al., 2018).

\begin{tabular}{|c|c|c|}
\hline$\underset{E}{\stackrel{D}{ٍ}}$ & Problèmes & Structures \\
\hline $\begin{array}{l}x \\
x \\
. \\
: \\
\Xi \\
\Xi\end{array}$ & $\begin{array}{l}\text { Problème } 1: \text { Club de Sport } \\
\text { Un Club de Sport propose trois types de jeux au cours de la } \\
\text { journée, sachant que chaque participant ait le droit de choisir } \\
\text { un seul jeu. Le nombre estimé de participants dans le } \\
\text { football est quatre fois le nombre de participants dans le } \\
\text { basket-ball et sept fois le nombre de participants au hand- } \\
\text { ball. Si vous savez que } 252 \text { personnes avaient participé au } \\
\text { football, quel est le nombre de participants dans chacun des } \\
\text { jeux, le hand-ball et le basket-ball? } \\
\text { Réponse : basket-ball: } 36 \text {, et hand-ball : } 63 \text {, total : } 351\end{array}$ & $\mathrm{X}$ \\
\hline 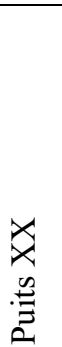 & $\begin{array}{l}\text { Problème } 2 \text { : Production de souliers } \\
\text { Une usine produit trois types de souliers. Le nombre de } \\
\text { souliers fabriqué pour les enfants est de } 7 \text { fois de celui } \\
\text { fabriqué pour les hommes et } 4 \text { fois le nombre de souliers } \\
\text { pour les femmes. Si cette usine produit un total de } 429 \\
\text { souliers par jour. Quel est le nombre de chaque type de } \\
\text { souliers produit par jour? } \\
\text { Réponse : hommes } 44 \text { : , enfants : } 308 \text {, femmes: } 77\end{array}$ & $\mathrm{X}$ \\
\hline 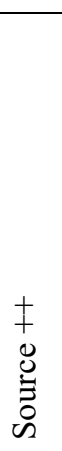 & $\begin{array}{l}\text { Problème } 3 \text { : Dépense d'une famille } \\
\text { Une famille dépense par mois, dans l'enseignement, } 870 \mathrm{dh} \\
\text { de plus que les frais de l'alimentation de ses enfants. Elle } \\
\text { dépense aussi, sur l'habillement } 850 \mathrm{dh} \text { de plus que les frais } \\
\text { d'alimentation. Elle compte un total de dépenses par mois } \\
\text { de } 4960 \mathrm{dh} \text {, combien dépense-t-elle dans l'alimentation, } \\
\text { l'enseignement de ses enfants et l'habillement? } \\
\text { Réponse : Alimentation: } 1080 \mathrm{dh} \text {; Enseignement des } \\
\text { enfants :1950dh; l'habillement : } 1930 \mathrm{dh}\end{array}$ & \\
\hline 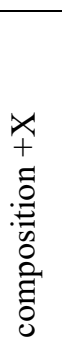 & $\begin{array}{l}\text { Problème } 4 \text { : Partage d'argent } \\
\text { Trois personnes partagent une somme d'argent. La part de } \\
\text { Khalid est de } 27 \text { dh de plus que celui de Tariq et que la part } \\
\text { de Samir est } 4 \text { fois plus que celui de Khalid. Si au total ils } \\
\text { ont } 441 \text { dh, combien ont-ils chacun? } \\
\text { Réponse : Samir : } 312 \text {, Khalid : } 78 \text {, Tariq : } 51\end{array}$ & \\
\hline
\end{tabular}




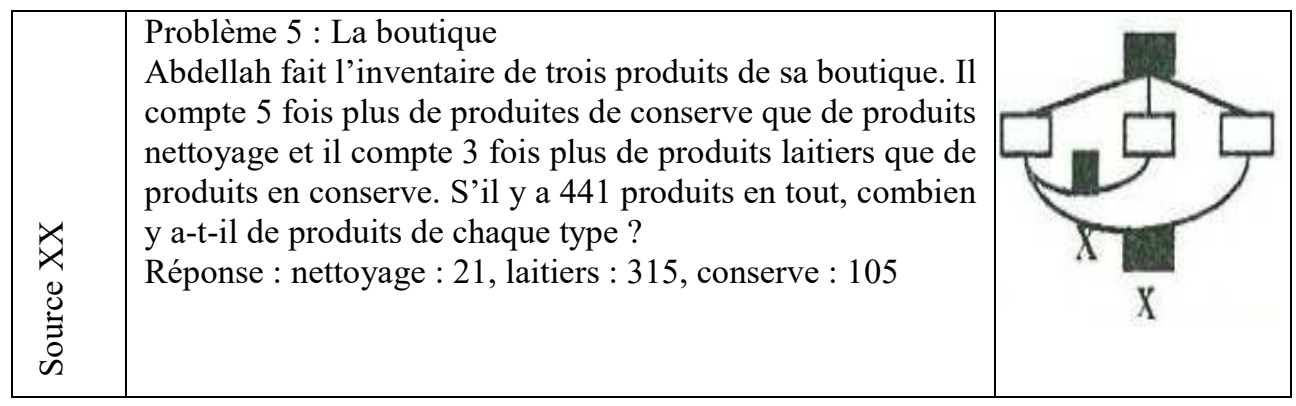

Annexe 2 : Les niveaux scolaires et tranches d'âges relatifs au système scolaire Marocain

\begin{tabular}{|c|c|c|c|c|c|c|c|c|c|c|c|}
\hline & \multicolumn{4}{|l|}{ Préscolaire } & \multicolumn{4}{|c|}{ Primaire } & \multicolumn{3}{l|}{$\begin{array}{l}\text { Secondaire } \\
\text { collégial }\end{array}$} \\
\hline $\begin{array}{c}3 \\
\text { à } 4\end{array}$ & $\begin{array}{c}4-5 \\
\text { ans }\end{array}$ & $\begin{array}{c}5-6 \\
\text { ans }\end{array}$ & $\begin{array}{c}6-7 \\
\text { ans }\end{array}$ & $\begin{array}{c}7- \\
8 \\
\text { ans }\end{array}$ & $\begin{array}{c}8- \\
9 \\
\text { ans }\end{array}$ & $\begin{array}{c}9- \\
10 \\
\text { ans }\end{array}$ & $\begin{array}{c}10- \\
11 \\
\text { ans }\end{array}$ & $\begin{array}{c}11- \\
12 \\
\text { ans }\end{array}$ & $\begin{array}{c}12-13 \\
\text { ans }\end{array}$ & $\begin{array}{c}13- \\
14 \\
\text { ans }\end{array}$ & $\begin{array}{c}14- \\
15 \\
\text { ans }\end{array}$ \\
\hline $\begin{array}{l}\text { Petite } \\
\text { Section }\end{array}$ & $\begin{array}{l}\text { Moyenne } \\
\text { Section }\end{array}$ & $\begin{array}{c}\text { Grande } \\
\text { Section }\end{array}$ & $\begin{array}{c}1 \text { ère } \\
\text { année }\end{array}$ & $2 \mathrm{e}$ & $3 \mathrm{e}$ & $4 \mathrm{e}$ & $5 \mathrm{e}$ & $6 \mathrm{e}$ & $\begin{array}{c}1 \mathrm{e} \\
\text { année }\end{array}$ & $2 \mathrm{e}$ & $3 \mathrm{e}$ \\
\end{tabular}

Les niveaux concernés 